\title{
Propagation of Waves at an Interface between a Liquid Half-Space and an Orthotropic Micropolar Solid Half-Space
}

\author{
Baljeet Singh $^{1}$ and Ritu Sindhu ${ }^{2}$ \\ ${ }^{1}$ Department of Mathematics, Post Graduate Government College, Sector 11, Chandigarh 160 011, India \\ ${ }^{2}$ Department of Mathematics, S. R. M. Institute of Engineering and Technology, Haryana, Khora-Bhura 134203, India
}

Correspondence should be addressed to Baljeet Singh,dr_baljeet@hotmail.com

Received 16 May 2011; Accepted 23 July 2011

Academic Editor: K. M. Liew

Copyright ( $) 2011$ B. Singh and R. Sindhu. This is an open access article distributed under the Creative Commons Attribution License, which permits unrestricted use, distribution, and reproduction in any medium, provided the original work is properly cited.

\begin{abstract}
An inviscid liquid half-space is considered in welded contact with a orthotropic micropolar solid half-space. Appropriate plane harmonic solutions of equations governing a liquid half-space and an orthotropic solid half-space are obtained. These solutions satisfy the required boundary conditions at the interface to obtain a system of four nonhomogeneous equations in amplitude ratios for incident quasi-longitudinal displacement wave. The amplitude ratios of various reflected and refracted waves are computed numerically for a particular example of the present model. The effect of anisotropy upon these amplitude ratios is shown graphically for a particular range of the angle of incidence.
\end{abstract}

\section{Introduction}

Material response to external stimuli depends heavily on the motions of its inner structures. Classical elasticity does not include this effect, where only translation degrees of freedom of material point of body is considered. Eringen [1] developed the linear micropolar theory of elasticity, which included the intrinsic rotations of the microstructure. It provides a model which can support body and surface couples and display high frequency optical branch of the wave spectrum. For engineering applications, it can model composites with rigid chopped fibres, elastic solid with rigid granular inclusions, and other industrial materials such as liquid crystals.

Parfitt and Eringen [2] investigated the plane wave propagation in an infinite isotropic homogeneous micropolar elastic solid half-space and showed the existence of four basic waves (a longitudinal displacement wave, a longitudinal microrotational wave, and two sets of two coupled waves) propagating with different velocities in an isotropic micropolar elastic solid. Smith [3] and Ariman [4] also studied the wave propagation in micropolar elastic solids.

The assumptions of isotropy in the solid medium may not capture some significant features of the continuum responses of soils, geological materials, and composites. Iesan [57] studied some static problems in orthotropic micropolar elastic solids. Kumar and Choudhary $[8,9]$ studied the mechanical sources and dynamic behaviour of orthotropic micropolar elastic medium. Kumar and Chaudhary [10] explained the plane strain problem in a homogeneous micropolar orthotropic elastic solids. Kumar and Ailawalia [11] studied the response of a micropolar cubic crystal due to various sources. Kumar and Gupta [12] studied the propagation of waves in transversely isotropic micropolar generalized thermoelastic half-space. Singh [13] investigated the two-dimensional plane wave propagation in an orthotropic micropolar elastic soli and showed the existence of three types of coupled plane waves in $x y$-plane, whose velocities depend upon the angle of propagation and material parameters. He also studied the reflection of these plane waves from a stress-free free surface and obtained the reflection coefficients for various reflected waves.

The study of wave motions at liquid-solid interface has been a topic of research for the last many years. Recently, Singh [14] studied the reflection and transmission of plane harmonic waves at an interface between liquid and micropolar viscoelastic solid with stretch, where the effects of micropolarity, viscosity, and stretch are observed on 
reflection and transmission coefficients. In the present paper, we have considered a problem on reflection and transmission at an interface between an inviscid liquid half-space and an orthotropic solid half-space. The reflection and transmission coefficients are computed numerically for a particular model to observe the effect of orthotropy.

\section{Formulation of the Problem and Solution}

We consider a homogeneous and orthotropic medium of an infinite extent with Cartesian coordinate system $\left(x_{1}, x_{2}, x_{3}\right)$. We restrict our study to the plane strain parallel to $x_{1}-x_{2}$ plane, with the displacement vector $\mathbf{u}=\left(u_{1}, u_{2}, 0\right)$ and microrotation vector $\phi=\left(0,0, \phi_{3}\right)$. Following Eringen [15] and Iesan [5], the field equations for homogeneous and orthotropic micropolar solid in $x_{1}-x_{2}$-plane in absence of body forces and couples are given by

$$
\begin{gathered}
A_{11} u_{1,11}+\left(A_{12}+A_{78}\right) u_{2,12}+A_{88} u_{1,22}-K_{1} \phi_{3,2}=\rho \ddot{u}_{1}, \\
\left(A_{12}+A_{78}\right) u_{1,12}+A_{77} u_{2,11}+A_{22} u_{2,22}-K_{2} \phi_{3,1}=\rho \ddot{u}_{2}, \\
B_{66} \phi_{3,11}+B_{44} \phi_{3,22}-\chi \phi_{3}+K_{1} u_{1,2}+K_{2} u_{2,1}=\rho j \ddot{\phi}_{3},
\end{gathered}
$$

where $A_{11}, A_{12}, A_{22}, A_{77}, A_{78}, A_{88}, B_{44}, B_{66}$ are characteristic constants of the material, $\rho$ is the density of the medium, and $j$ is the microinertia. Here, $\chi=K_{2}-K_{1}, K_{1}=A_{78}-$ $A_{88}, K_{2}=A_{77}-A_{78}, \ddot{u}_{i}=\left(\partial^{2} u_{i} / \partial t^{2}\right), u_{i, j k}=\left(\partial^{2} u_{i} / \partial x_{j} \partial x_{k}\right)$, and $\ddot{\phi}_{i}=\left(\partial^{2} \phi_{i} / \partial t^{2}\right)$.

Solutions of (1) to (3) are now sought in the form of the harmonic travelling wave:

$$
\left(u_{i}, \phi_{3}\right)=\left(A d_{i}, k B\right) e^{i k\left(x_{1} p_{1}+x_{2} p_{2}-v t\right)}, \quad(i=1,2),
$$

where $k$ is the wave number, $v$ is the phase velocity, $d_{i}$ are components of unit displacement vector, $p_{j}(j=1,2)$ are components of propagation vector, and $A, B$ are arbitrary constants.

Using (4), (1) to (3) reduce to

$$
\begin{aligned}
& \left(D_{1}-\rho v^{2}\right) A d_{1}+\left(A_{12}+A_{78}\right) p_{1} p_{2} A d_{2}+\iota p_{2} K_{1} B=0, \\
& \left(A_{12}+A_{78}\right) p_{1} p_{2} A d_{1}+\left(D_{2}-\rho v^{2}\right) A d_{2}+\iota p_{1} K_{2} B=0, \\
& p_{2} K_{1} A d_{1}+p_{1} K_{2} A d_{2}+\iota\left[\chi+j k^{2}\left(D_{3}-\rho v^{2}\right)\right] B=0,
\end{aligned}
$$

where $D_{1}=A_{11} p_{1}^{2}+A_{88} p_{2}^{2}, D_{2}=A_{77} p_{1}^{2}+A_{22} p_{2}^{2}$, and $D_{3}=\left(B_{66} p_{1}{ }^{2}+B_{44} p_{2}{ }^{2}\right) / j$. The nontrivial solution of (5) gives the following cubic equation in $\zeta$ :

$$
\zeta^{3}+L \zeta^{2}+M \zeta+N=0,
$$

where $\zeta=\rho v^{2}, L=-\left[D_{1}+D_{2}+D_{3}+\left(\chi / j k^{2}\right)\right], M=D_{1} D_{2}+$ $D_{2} D_{3}+D_{3} D_{1}+\left(D_{1}+D_{2}\right)\left(\chi / j k^{2}\right)-\left(1 / j k^{2}\right)\left(p_{1}^{2} K_{2}^{2}+p_{2}^{2} K_{1}^{2}\right)-$ $\left(A_{12}+A_{78}\right)^{2} p_{1}^{2} p_{2}^{2}, N=-\left[D_{1} D_{2} D_{3}+\left(\chi / j k^{2}\right)\left\{D_{1} D_{2}-\right.\right.$ $\left.\left(A_{12}+A_{78}\right)^{2} p_{1}^{2} p_{2}^{2}\right\}-\left(1 / j k^{2}\right)\left\{D_{1} K_{2}^{2} p_{1}^{2}+D_{2} K_{1}^{2} p_{2}^{2}-\right.$ $\left.\left.2 K_{1} K_{2}\left(A_{12}+A_{78}\right) p_{1}^{2} p_{2}^{2}\right\}-D_{3}\left(A_{12}+A_{78}\right)^{2} p_{1}^{2} p_{2}^{2}\right]$ The three roots $\zeta_{1}, \zeta_{2}$, and $\zeta_{3}$ of the cubic equation (6) correspond to three types of quasi-coupled waves, whose velocities depend upon the angle of propagation. Let us name these three waves as quasi-longitudinal displacement (QLD) wave, quasi coupled transverse microrotational (QCTM) wave and quasi coupled transverse displacement (QCTD) wave. The corresponding velocities of these waves are identified from computer program of the solution of (6). It is found that the velocities $v_{1}, v_{2}$, and $v_{3}$, correspond to QLD, QCTM, and QCTD waves, respectively [13].

If we put $A_{11}=A_{22}=\lambda+2 \mu+\kappa, A_{77}=A_{88}=$ $\mu+\kappa, A_{12}=\lambda, A_{78}=\mu,-K_{1}=K_{2}=\chi / 2=\kappa$, in (6), then the velocity $v_{1}$ corresponds to longitudinal displacement wave and the velocities $v_{2}, v_{3}$ correspond to two coupled transverse waves as obtained by Parfitt and Eringen [2] in the theory of isotropic and linear micropolar elasticity. These velocities do not depend upon the angle of propagation.

If we put $A_{77}=A_{88}=A_{78}=B_{44}=B_{66}=K_{1}=K_{2}=$ $\chi=0, A_{11}=A_{22}=A_{12}=\lambda^{\prime}$ in (6), the cubic equation (6) reduces to the linear equation $\rho^{\prime} v^{\prime 2}=\lambda^{\prime}$, which gives the velocity of longitudinal wave in the liquid medium. Here, the parameters with primes correspond to the liquid medium.

\section{Reflection and Transmission}

We consider a homogeneous and orthotropic micropolar medium and a liquid medium of an infinite extent with cartesian coordinate system $\left(x_{1}, x_{2}, x_{3}\right)$ having origin at the interface. The positive $x_{3}$-axis is pointing into the orthotropic micropolar solid half-space. The $x_{1}$-axis is along the interface between the liquid half-space and orthotropic micropolar solid half-space. In this section, we shall derive the relations between the reflection and transmission coefficients, when (QLD, QCTM, or QCTD) wave becomes incident at the interface $x_{2}=0$. For incident (QLD, QCTM, or QCTD) wave, there will be reflected QLD, QCTM, and QCTD waves in solid half-space and transmitted longitudinal wave in the liquid half-space as shown in Figure 1. Accordingly, if the wave normal to the incident wave through solid medium makes an angle $\theta_{0}$ with the positive direction of $x_{2}$-axis, then wave normals to the reflected QLD, QCTM, and QCTD and refracted longitudinal waves make $\theta_{1}, \theta_{2}$, and $\theta_{3}$ and and $\theta^{\prime}$ with $x_{2}$-axis. The required boundary conditions at the interface, $x_{2}=0$, are the continuity of normal force stresses, vanishing of tangential force stress, vanishing of tangential couple stress, and continuity of normal components of displacement vector, that is,

$$
t_{22}{ }^{(\alpha)}=t_{22}^{\prime}{ }^{(\alpha)}, \quad \begin{array}{r}
t_{21}{ }^{(\alpha)}=0, \\
u_{2}{ }^{(\alpha)}=u_{2}^{\prime}
\end{array}
$$

where

$$
\begin{aligned}
& t_{22}{ }^{(\alpha)}=A_{12} u_{1,1}+A_{22} u_{2,2}, \\
& t_{21}{ }^{(\alpha)}=A_{78} u_{2,1}+A_{88} u_{1,2}+\left(A_{88}-A_{78}\right) \phi_{3}, \\
& m_{23}{ }^{(\alpha)}=B_{44} \phi_{3,2}, \\
& t_{22}^{\prime}=\lambda^{\prime}\left(u_{1,1}^{\prime}+u_{2,2}^{\prime}\right) .
\end{aligned}
$$




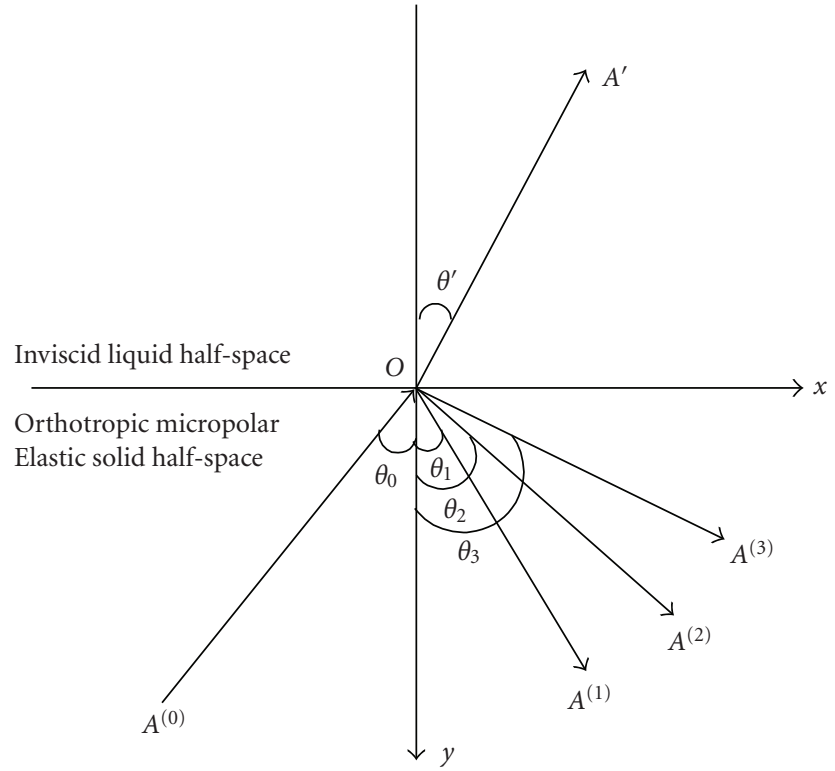

Figure 1: Geometry of the problem.

The following components of displacement and microrotation vectors are appropriate to satisfy the boundary conditions (7)

$$
\begin{gathered}
u_{1}{ }^{(\alpha)}=A^{(\alpha)} d_{1}^{(\alpha)} e^{i \eta_{\alpha}}, \\
u_{2}^{(\alpha)}=G^{(\alpha)} A^{(\alpha)} d_{1}^{(\alpha)} e^{i \eta_{\alpha}}, \\
\phi_{3}^{(\alpha)}=i k_{\alpha} H^{(\alpha)} A^{(\alpha)} d_{1}^{(\alpha)} e^{i \eta_{\alpha}}, \\
u_{2}^{\prime}=G^{\prime} A^{\prime} d_{1}^{\prime} e^{i \eta^{\prime}} .
\end{gathered}
$$

Here, $\eta_{\alpha}=k_{\alpha}\left(x p_{1}{ }^{(\alpha)}+y p_{2}{ }^{(\alpha)}-v_{\alpha} t\right), \alpha=0$ corresponds to incident wave; $\alpha=1,2,3$ correspond to reflected waves, and

$$
\begin{gathered}
G^{(\alpha)}=\frac{K_{1}\left(A_{12}+A_{78}\right) p_{1}{ }^{(\alpha)} p_{2}{ }^{(\alpha)^{2}}-K_{2} p_{1}{ }^{(\alpha)}\left(D_{1}{ }^{(\alpha)}-\rho v_{\alpha}{ }^{2}\right)}{K_{2}\left(A_{12}+A_{78}\right) p_{2}{ }^{(\alpha)} p_{1}{ }^{(\alpha)^{2}}-K_{1} p_{2}{ }^{(\alpha)}\left(D_{2}{ }^{(\alpha)}-\rho v_{\alpha}{ }^{2}\right)}, \\
H^{(\alpha)}=\frac{K_{1} p_{2}{ }^{(\alpha)}+K_{2} G^{(\alpha)} p_{1}{ }^{(\alpha)}}{\chi+j k^{2}\left(D_{3}{ }^{(\alpha)}-\rho v_{\alpha}{ }^{2}\right)}
\end{gathered}
$$

where

$$
\begin{gathered}
D_{1}^{(\alpha)}=A_{11} p_{1}{ }^{(\alpha)^{2}}+A_{88} p_{2}{ }^{(\alpha)^{2}}, \\
D_{2}{ }^{(\alpha)}=A_{77} p_{1}{ }^{(\alpha)^{2}}+A_{22} p_{2}{ }^{(\alpha)^{2}}, \\
D_{3}{ }^{(\alpha)}=\frac{\left(B_{66} p_{1}{ }^{(\alpha)^{2}}+B_{44} p_{2}{ }^{(\alpha)^{2}}\right)}{j},
\end{gathered}
$$

and $G^{\prime}$ and $\eta^{\prime}$ correspond to the liquid medium.
With the help of above displacement and microrotation components, the boundary conditions (7) result into the following three relations

$$
\begin{gathered}
k_{\alpha}\left[A_{12} p_{1}{ }^{(\alpha)}+A_{22} G^{(\alpha)} p_{2}{ }^{(\alpha)}\right] A^{(\alpha)} d_{1}^{(\alpha)} e^{i k_{\alpha}\left(x p_{1}^{(\alpha)}-v_{\alpha} t\right)} \\
-k^{\prime} \lambda^{\prime}\left[p_{1}^{\prime}+G^{\prime} p_{2}^{\prime}\right] A^{\prime} d_{1}^{\prime} e^{i k^{\prime}\left(x_{1} p_{1}^{\prime}-v^{\prime} t\right)}=0, \\
{\left[A_{78} p_{1}{ }^{(\alpha)} G^{(\alpha)}+A_{88} p_{2}^{(\alpha)}+\left(A_{88}-A_{78}\right) H^{(\alpha)}\right]} \\
\times k_{\alpha} A^{(\alpha)} d_{1}^{(\alpha)} e^{i k_{\alpha}\left(x p_{1}^{(\alpha)}-v_{\alpha} t\right)}=0, \\
k_{\alpha}{ }^{2} H^{(\alpha)} p_{2}{ }^{(\alpha)} A^{(\alpha)} d_{1}{ }^{(\alpha)} e^{i k_{\alpha}\left(x p_{1}{ }^{(\alpha)}-v_{\alpha} t\right)}=0, \\
G^{(\alpha)} A^{(\alpha)} d_{1}^{(\alpha)} e^{i k_{\alpha}\left(x_{1} p_{1}{ }^{(\alpha)}-v_{\alpha} t\right)}-G^{\prime} A^{\prime} d_{1}^{\prime} e^{i k^{\prime}\left(x_{1} p_{1}^{\prime}-v^{\prime} t\right)}=0 .
\end{gathered}
$$

In view of the fact that (12) must hold for all $x$ and $t$, it is deduced that

$$
\begin{gathered}
k_{0} p_{1}^{(0)}=k_{1} p_{1}{ }^{(1)}=k_{2} p_{1}{ }^{(2)}=k_{3} p_{1}{ }^{(3)}=k^{\prime} p_{1}{ }^{\prime} \equiv k, \\
k_{0} v_{0}=k_{1} v_{1}=k_{2} v_{2}=k_{3} v_{3}=k^{\prime} v^{\prime} \equiv \omega,
\end{gathered}
$$

From (12), the analytical expressions $Z_{1}=A^{(1)} / A^{(0)}, Z_{2}=$ $A^{(2)} / A^{(0)}, Z_{3}=A^{(3)} / A^{(0)}$, and $Z_{4}=A^{\prime} / A^{(0)}$ for reflection coefficients of QLD, QCTM, and QCTD waves and transmission coefficient of longitudinal wave may be obtained for further numerical computations.

\section{Numerical Example}

The following relevant physical constants are chosen arbitrarily for a composite as an orthotropic micropolar material due to unavailability of relevant experimental data for such material in literature [8].

$A_{11}=11.65 \times 10^{10} \mathrm{~N} \mathrm{~m}^{-2}, A_{22}=11.71 \times 10^{10} \mathrm{~N} \mathrm{~m}^{-2}$, $A_{12}=7.69 \times 10^{10} \mathrm{~N} \mathrm{~m}^{-2}, \quad A_{77}=1.9959 \times 10^{10} \mathrm{~N} \mathrm{~m}^{-2}, A_{78}=$ $1.98 \times 10^{10} \mathrm{~N} \mathrm{~m}^{-2}, A_{88}=2.0159 \times 10^{10} \mathrm{~N} \mathrm{~m}^{-2}, B_{44}=$ $0.036 \times 10^{10} \mathrm{~N}, B_{66}=0.037 \times 10^{10} \mathrm{~N}, \rho=2190 \mathrm{~kg} \mathrm{~m}^{-3}, j=$ $0.000196 \mathrm{~m}^{2}, j k^{2}=1$. The following physical constants for aluminium-epoxy composite as an isotropic micropolar elastic solid are considered [16]: $\rho=2190 \mathrm{~kg} \mathrm{~m}^{-3}, \lambda=$ $7.59 \times 10^{10} \mathrm{~N} \mathrm{~m}^{-2}, \quad \mu=1.89 \times 10^{10} \mathrm{~N} \mathrm{~m}^{-2}, \kappa=0.0149 \times$ $10^{10} \mathrm{~N} \mathrm{~m}^{-2}, \gamma=0.0268 \times 10^{10} \mathrm{~N}, j=0.000196 \mathrm{~m}^{2}, j k^{2}=1$.

The following physical constants for inviscid liquid are considered $\rho^{\prime}=1000 \mathrm{~kg} \mathrm{~m}^{-3}, v^{\prime}=1.435 \times 10^{4} \mathrm{~m} \mathrm{~s}^{-1}$,

For the incidence of QLD wave, the complex absolute values of the reflection and transmission coefficients are computed numerically with the help of above physical constants of orthotropic and isotropic micropolar materials with the following components of the propagation and unit displacement vectors:

$$
\begin{gathered}
p_{1}{ }^{(0)}=\sin \theta_{0}, \quad p_{2}{ }^{(0)}=\cos \theta_{0}, \\
p_{1}{ }^{(1)}=\sin \theta_{1}, \quad p_{2}{ }^{(1)}=-\cos \theta_{1}, \\
d_{1}{ }^{(0)}=\cos \theta_{0}, \quad d_{2}{ }^{(0)}=-\sin \theta_{0}, \\
d_{1}{ }^{(1)}=\cos \theta_{1}, \quad d_{2}{ }^{(1)}=\sin \theta_{1},
\end{gathered}
$$



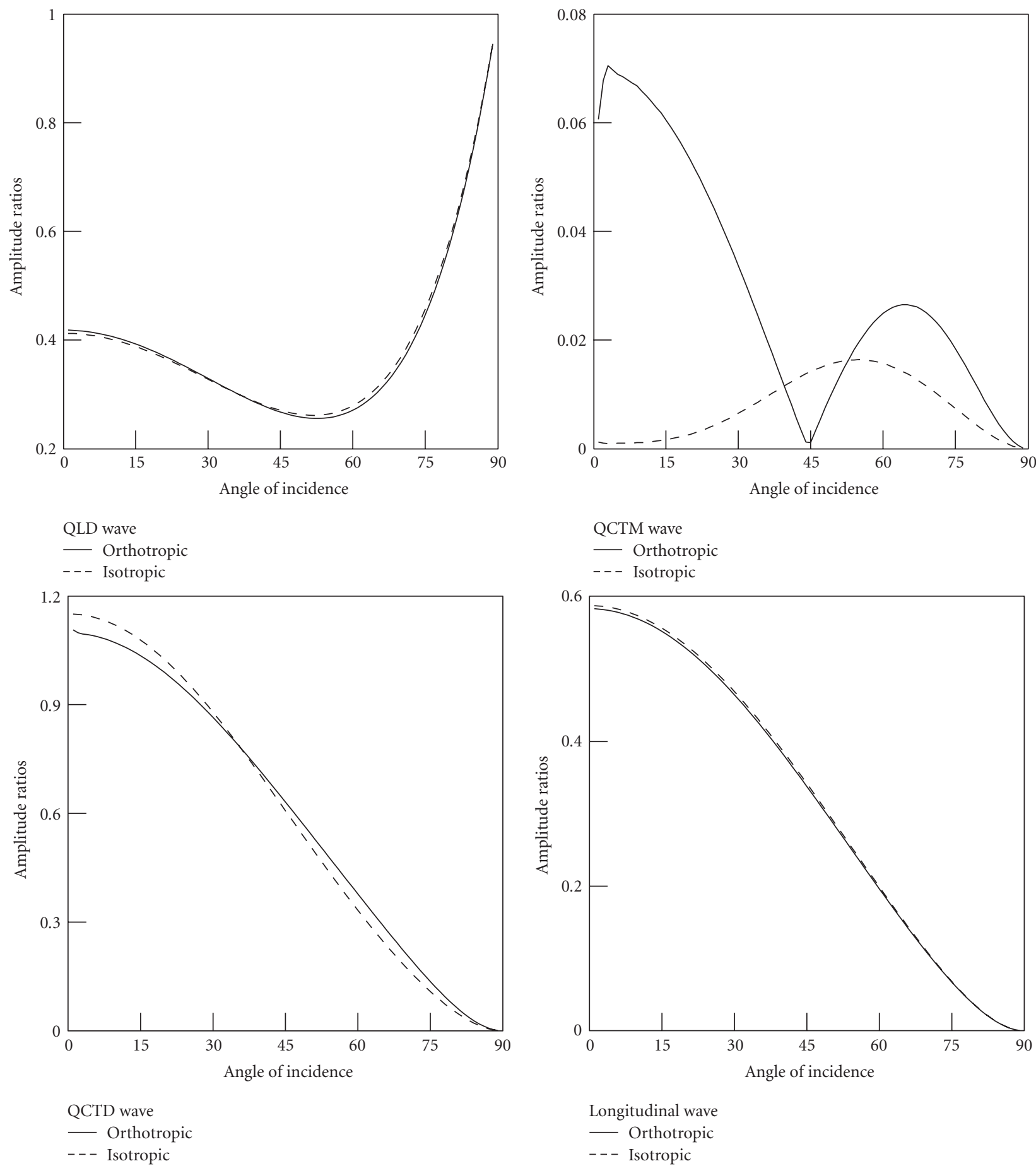

FIGURE 2: Variations of amplitude ratios of reflected QLD, QCTM, QCTD and refracted longitudinal waves against the angle of incidence.

$$
\begin{aligned}
p_{1}{ }^{(2)}=\sin \theta_{2}, & p_{2}{ }^{(2)}=-\cos \theta_{2}, \\
p_{1}{ }^{(3)}=\sin \theta_{3}, & p_{2}{ }^{(3)}=-\cos \theta_{3}, \\
d_{1}{ }^{(2)}=\cos \theta_{2}, & d_{2}{ }^{(2)}=\sin \theta_{2}, \\
d_{1}{ }^{(3)}=\cos \theta_{3}, & d_{2}{ }^{(3)}=\sin \theta_{3}, \\
p_{1}^{\prime}=\sin \theta^{\prime}, & p_{2}^{\prime}=\cos \theta^{\prime},
\end{aligned}
$$$$
d_{1}^{\prime}=\cos \theta^{\prime}, \quad d_{2}^{\prime}=-\sin \theta^{\prime} .
$$

The reflection coefficient $Z_{1}$ of reflected QLD wave has value 0.4182 near normal incidence. It decreases to its mini-mum value 0.2554 at $\theta_{0}=53^{\circ}$; thereafter, it increases to its maximum value 0.9447 near grazing incidence. The reflection coefficient $Z_{2}$ of reflected QCTM wave has value 0.0606 
near normal incidence. Initially, it increases to its maximum value 0.0705 at $\theta_{0}=3^{\circ}$, but then it sharply decreases to 0.0011 at $\theta_{0}=45^{\circ}$. Thereafter, it increases to value 0.0265 at $\theta_{0}=65^{\circ}$, and then decreases to its minimum value 0.0001 at $\theta_{0}=89^{\circ}$. The reflection coefficient $Z_{3}$ of QCTD wave has its maximum value 1.1057 at $\theta_{0}=1^{\circ}$. It decreases slowly to its minimum value 0.0009 near grazing incidence. The transmission coefficient $Z_{4}$ of longitudinal wave has its maximum value 0.5837 at $\theta_{0}=1^{\circ}$. It decreases slowly to its minimum value 0.0004 near grazing incidence. These variations of reflection and transmission coefficients are shown in Figure 2 by solid lines. The dotted lines in Figure 2 correspond to the variations for isotropic micropolar case. On comparing the solid and dotted lines, it is observed that the reflection coefficients of reflected QCTM and QCTD waves are significantly affected due to the presence of orthotropy in micropolar medium. The coefficients of reflected QLD and refracted longitudinal waves are also affected slightly due to the anisotropy in the solid medium.

\section{Concluding Remarks}

The relevant boundary conditions at an interface between the liquid half-space and orthotropic micropolar solid half-space interface are satisfied by appropriate solutions in the both half-spaces to obtain the relations between reflection and transmission coefficients of various reflected and refracted waves for the incidence of QLD, QCTM, or QCTD wave. The numerical computations are carried out for the incidence of QLD wave only. The dependence of reflection and transmission coefficients on the angle of incidence is shown graphically to observe the effect of orthotropy in solid medium. The present numerical study might provide more relevant information about the wave propagation in orthotropic material if we had relevant experimental physical data for an orthotropic micropolar material. The present theoretical and numerical analysis may be helpful to experimental seismologists working in the fields of wave propagation in solids.

\section{References}

[1] A. C. Eringen, "Linear Theory of Micropolar Elasticity," Journal of Mathematics and Mechanics, vol. 15, pp. 909-924, 1966.

[2] V. R. Parfitt and A. C. Eringen, "Reflection of plane waves from a flat boundary of a micropolar elastic half-space," Journal of the Acoustical Society of America, vol. 45, no. 5, pp. 1258-1272, 1971.

[3] A. C. Smith, "Waves in micropolar elastic solids," International Journal of Engineering Science, vol. 5, no. 10, pp. 741-746, 1967.

[4] T. Ariman, "Wave propagation in a micropolar elastic halfspace," Acta Mechanica, vol. 13, no. 1-2, pp. 11-20, 1972.

[5] D. Iesan, "The plane micropolar strain of orthotropic elastic solids," Archives of Mechanics, vol. 25, no. 3, pp. 547-561, 1973.

[6] D. Iesan, "Torsion of anisotropic elastic cylinders," Zeitschrift für Angewandte Mathematik und Mechanik, vol. 54, no. 12, pp. 773-779, 1974.
[7] D. Iesan, "Bending of orthotropic micropolar elastic beams by terminal couples," Analele Stiintifice ale Universitatii "Al. I. Cuza" din Iasi, vol. 25, pp. 411-418, 1974.

[8] R. Kumar and S. Choudhary, "Mechanical sources in orthotropic micropolar continua," Proceedings of the Indian Academy of Sciences, Earth and Planetary Sciences, vol. 111, no. 2, pp. 133-141, 2002.

[9] R. Kumar and S. Choudhary, "Dynamical behaviour of orthotropic micropolar elastic medium," JVC/Journal of Vibration and Control, vol. 8, no. 8, pp. 1053-1069, 2002.

[10] R. Kumar and S. Choudhary, "Response of orthotropic micropolar elastic medium due to time harmonic source," vol. 29, no. 1, pp. 83-92.

[11] R. Kumar and P. Ailawalia, "Deformation in micropolar cubic crystal due to various sources," International Journal of Solids and Structures, vol. 42, no. 23, pp. 5931-5944, 2005.

[12] R. Kumar and R. R. Gupta, "Propagation of waves in transversely isotropic micropolar generalized thermoelastic half space," International Communications in Heat and Mass Transfer, vol. 37, no. 10, pp. 1452-1458, 2010.

[13] B. Singh, "Wave propagation in an orthotropic micropolar elastic solid," International Journal of Solids and Structures, vol. 44, no. 11-12, pp. 3638-3645, 2007.

[14] B. Singh, "Reflection and transmission of plane harmonic waves at an interface between liquid and micropolar viscoelastic solid with stretch," Sadhana, vol. 25, no. 6, pp. 589-600, 2000.

[15] A. C. Eringen, "Theory of micropolar elasticity," in Fracture, vol. 2, pp. 621-729, Academic Press, 1968.

[16] R. D. Gauthier, "Experimental investigation on micropolar media," in Mechanics of Micropolar Media, O. Brulin and R. K. T. Hsieh, Eds., World Scientific, Singapore, 1982. 

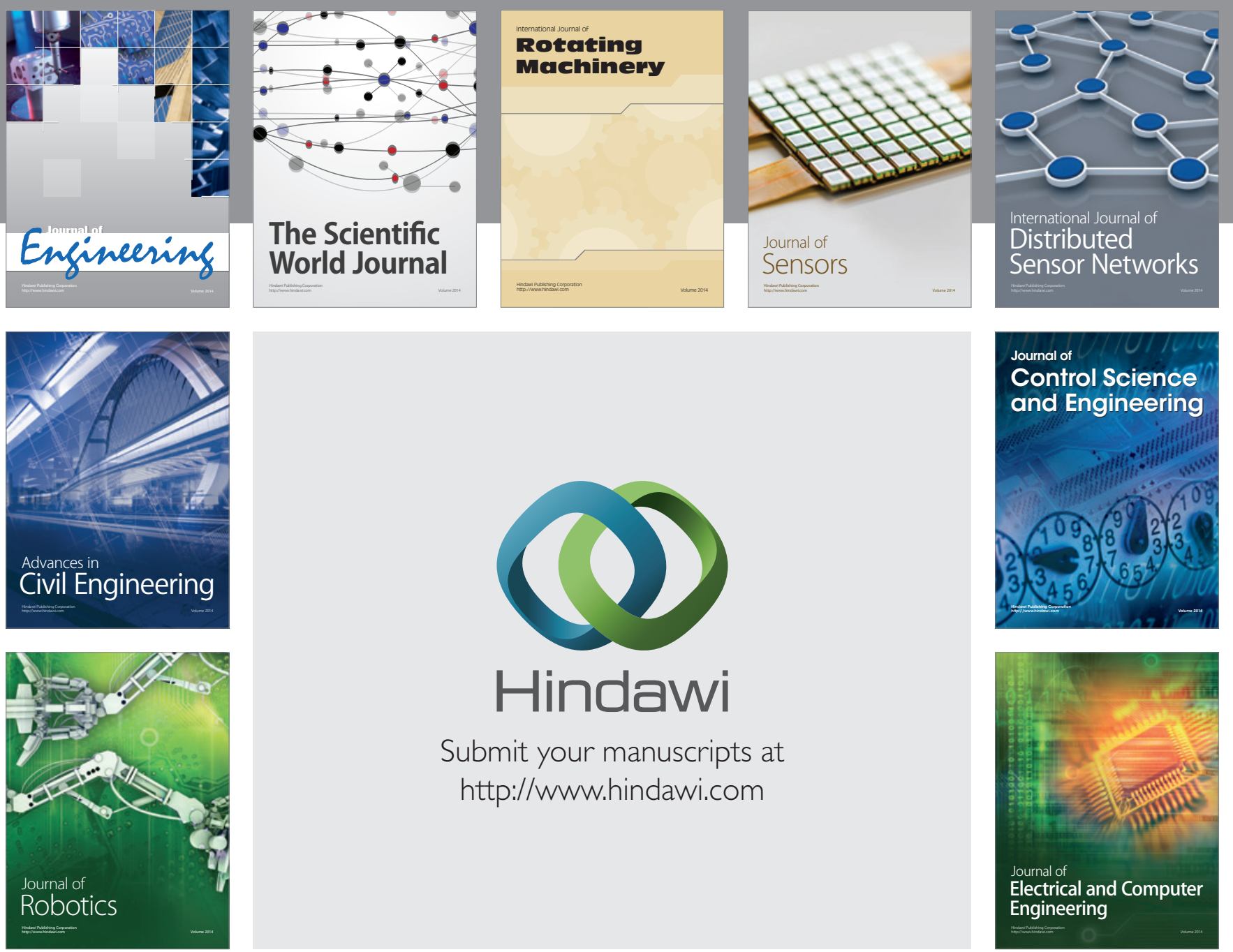

Submit your manuscripts at

http://www.hindawi.com
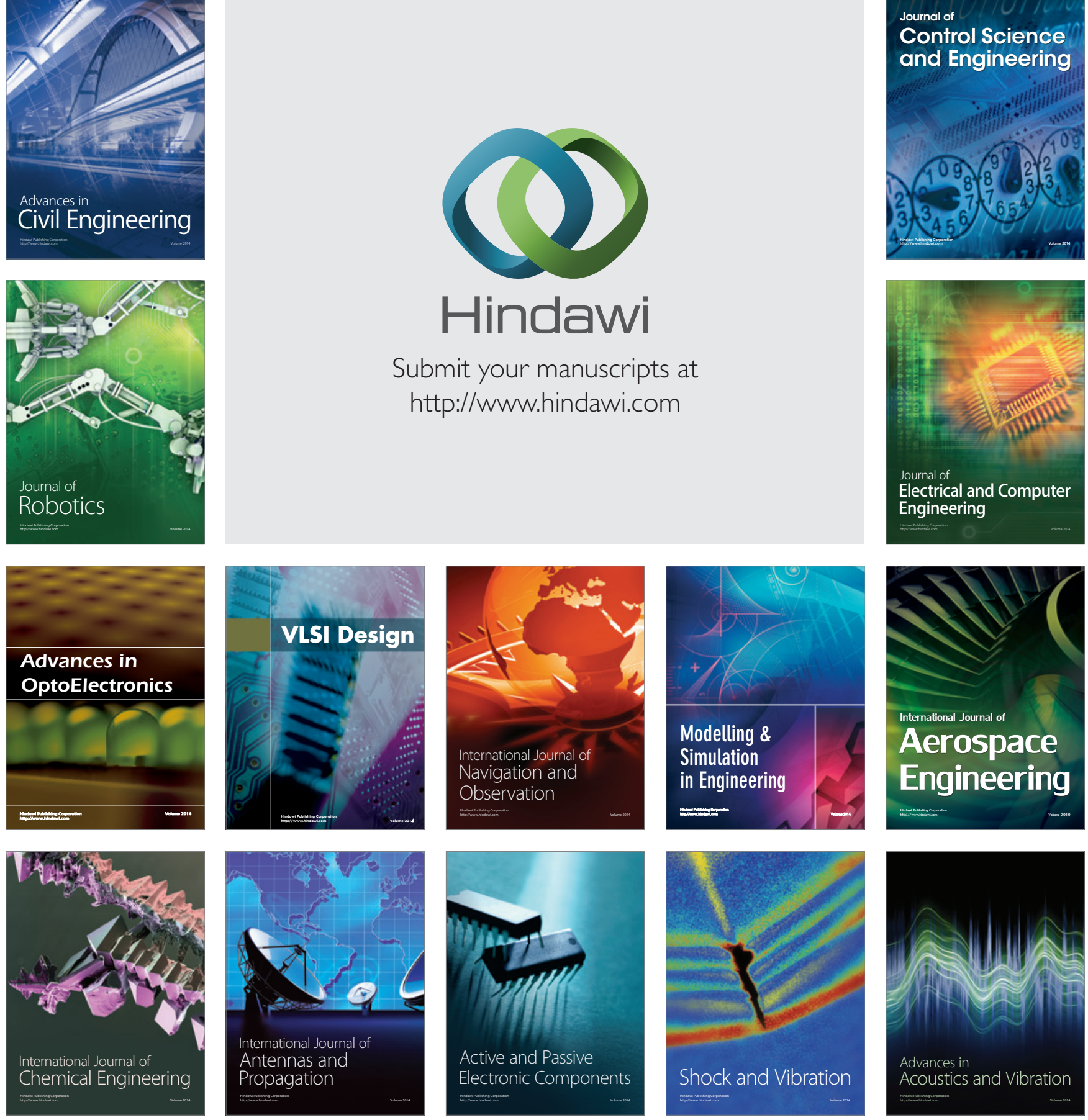